\title{
A Particle Swarm Algorithm for Symbols Detection in Wideband Spatial Multiplexing Systems
}

\author{
A. A. Khan \\ Centre for Advanced Studies in \\ Engineering \\ Islamabad, Pakistan \\ +923215204266 \\ adkhan100@gmail.com
}

\author{
M. Naeem \\ Simon Fraser University Burnaby \\ BC Canada \\ mnaeem@sfu.ca
}

\author{
S. I. Shah \\ Iqra University Islamabad Campus \\ Pakistan \\ ismail@iqraisb.edu.pk
}

\begin{abstract}
This paper explores the application of the particle swarm algorithm for a NP-hard problem in the area of wireless communications. The specific problem is of detecting symbols in a Multi-Input Multi-Output (MIMO) communications system. This approach is particularly attractive as PSO is well suited for physically realizable, real-time applications, where low complexity and fast convergence is of absolute importance. While an optimal Maximum Likelihood (ML) detection using an exhaustive search method is prohibitively complex, we show that the Swarm Intelligence (SI) optimized MIMO detection algorithm gives near-optimal Bit Error Rate (BER) performance in fewer iterations, thereby reducing the ML computational complexity significantly. The simulation results suggest that the proposed detector gives an acceptable performance complexity trade-off in comparison with ML and VBLAST detectors.
\end{abstract}

\section{Categories and Subject Descriptors}

C.2.1 [Computer-Communication Networks]: Network Architecture and Design - Wireless communication.

\section{General Terms}

Algorithms, Performance.

\section{Keywords}

PSO, Multi-Input Multi-Output systems, and symbol detection.

\section{INTRODUCTION}

Real life optimization problems are often so complex that finding the best solution becomes computationally infeasible. Therefore, an intelligent approach is to search for a good approximate solution consuming lesser computational resources. Several engineering problems contain multiple objectives that need to be addressed simultaneously. Many techniques have been proposed that imitate nature's own ingenious ways to explore optimal solutions for both single and multi-objective optimization

Permission to make digital or hard copies of all or part of this work for personal or classroom use is granted without fee provided that copies are not made or distributed for profit or commercial advantage and that copies bear this notice and the full citation on the first page. To copy otherwise, or republish, to post on servers or to redistribute to lists, requires prior specific permission and/or a fee.

GECCO'07, July 7-11, 2007, London, England, United Kingdom. Copyright 2007 ACM 978-1-59593-697-4/07/0007...\$5.00. problems. Earliest of the nature inspired techniques are genetic and other evolutionary heuristics that evoke Darwinian evolution principles.

Particle Swarm Optimization (PSO) meta-heuristic is a population-based search algorithm, inspired by the coordinated movements of birds flocking introduced by Kennedy and Eberhart in 1995 [1],[2]. Standard PSO uses a real-valued multidimensional solution space [1], whereas in binary PSO particle positions are discrete rather than real valued [3]. Its simple mathematical model, resistance to being trapped in local minima and convergence to near optimal solution in fewer iterations makes it a suitable candidate for real-time NP-hard communication problems [4], in addition to other wide range of applications [5].

In this paper, PSO's binary version is applied to a NP-hard problem in the area of wireless communications. The problem is to detect symbols from a composite signal, received at multiple receivers, transmitted from multiple transmitters. This MIMO detection problem is one of the most important issues faced in wireless communications area.

The relevant information-theoretic analysis reveals that significant performance gains are achievable in wireless communication systems using a MIMO architecture employing multiple antennas [6]. This architecture is suitable for higher data rate multimedia communications [7]. Efficient exploitation of spatial diversity available in MIMO channel enables higher system capacity. Orthogonal Frequency Division Multiplexing (OFDM) employed in conjunction with MIMO architecture constitutes an attractive solution for modern wireless communications systems [8]. One of the challenges in building wide band MIMO systems is the tremendous processing power required at the receiver side. While coded MIMO schemes offer better performance than separate channel coding and modulation scheme by fully exploring the tradeoff between multiplexing and diversity [9], its hardware complexity can be significant, especially for wide band system with more than four antennas both at the transmitter and the receiver sides. On the other hand, it is easier to implement traditional channel coding schemes such as Convolution code and Turbo code for data rates of hundreds of Mbps. For this reason we discuss uncoded MIMO system also called spatial multiplexing as shown in Figure 1. 


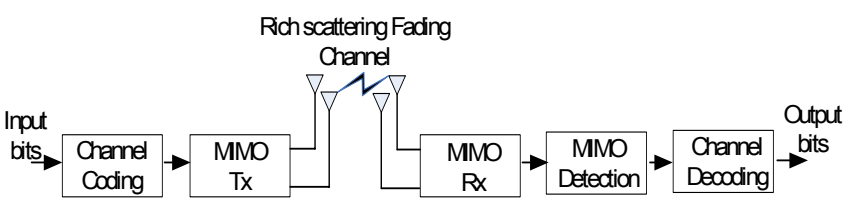

Figure 1: Spatial Multiplexing System

MIMO symbol detection involves decoding of complex signals at the receiver, which is considerably complicated than Single-Input Single-Output (SISO) case, because of multiple transmitters and receivers. Simultaneous transmission of signals from multiple transmitters complicates the detection of composite signal at the receiver.

There are many MIMO detection technologies for spatial multiplexing [10]. These techniques can be divided into linear and non-linear detection methods. Linear methods offer low complexity with degraded performance as compared to non-linear methods. This paper focuses on non-linear detectors and makes an effort to improve performance at the cost of complexity and vice versa. Maximum Likelihood (ML) and Vertical Bell labs Layered Space Time (V-BLAST) detectors [11],[12] are two famous nonlinear MIMO detection methods. Both of these non-linear detectors have their own advantages. ML outperforms VBLAST, while VBLAST possess low complexity than ML. Previous work [13],[14] tried to take advantage of these two methods and the results show a performance complexity trade off between the two methods.

Worst-case complexity of computing the exact ML solution is generically exponential, due to NP-hardness [10]. ML detection in MIMO communication system belongs to a large class of combinatorial problem known as NP-hard optimization problems. Therefore, in order to solve these NP-hard problem for any nontrivial problem size, one of the following approaches can be used: (1) Approximation: An algorithm which quickly finds a suboptimal solution which is within a certain range of the optimal one; (2) Probabilistic: An algorithm which provably yields good average runtime behavior for a given distribution of the problem instances; and (3) Heuristic: An algorithm which works "reasonably well" on many cases, but for which there is no proof that it is always fast (e.g., evolutionary techniques).

We report a Binary PSO assisted MIMO detection algorithm with a reasonable performance complexity tradeoff and to the best of authors understanding this is first successful attempt to optimize MIMO detection using PSO meta-heuristics.

The rest of the paper is organized as follows. Section-2 provides the wideband spatial multiplexing system model and sets-up MIMO detection problem for flat-fading channels. A brief overview of existing MIMO detectors is given in section-3. Section-4 details the proposed detection algorithm. Performance of the proposed detector is reported in next sections followed by conclusions.

\section{MIMO DETECTION FOR FLAT- FADING CHANNEL}

\subsection{MIMO Channel Model}

Consider a MIMO system where $N_{t}$ different signals are transmitted and arrive at an array of $N_{r}\left(N_{t} \leq N_{r}\right)$ receivers via a rich-scattering flat-fading environment. Grouping all the transmitted and received signals into vectors, the system can be viewed as transmitting an $N_{t} \times 1$ vector signal $\mathbf{x}$ through an $N_{t} \mathrm{x} N_{r}$ matrix channel $\mathbf{H}$, with $N_{r} \times 1$ Gaussian noise vector $\mathbf{v}$ added at the input of the receiver.

$$
\mathbf{y}=\mathbf{H x}+\mathbf{v}
$$

where $\mathbf{y}$ is the received $N_{r} \times 1$ vector. The $\left(n_{r}, n_{t}\right)^{\text {th }}$ element of $\mathbf{H}$, $h_{n_{r} n_{t}}$, is the complex channel response from the $n_{t}^{\text {th }}$ transmit antenna to the $n_{r}^{\text {th }}$ receive antenna. $\mathbf{x}$ is zero mean and has covariance matrix of $\mathbf{R}_{\mathbf{x}}=\mathrm{E}\{\mathbf{x x} *\}=\sigma_{x}^{2} \mathbf{I}$. The vector $\mathbf{v}$ is also zero-mean and $\mathbf{R}_{\mathbf{v}}=\mathrm{E}\left\{\mathbf{\mathbf { v v } ^ { * } \}}=\sigma_{v}^{2} \mathbf{I}\right.$. In frequency-selective fading channels, the entire channel frequency response $h_{n_{r} n_{t}}$ is no longer characterized by a constant, but rather a function of the frequency.

$$
\mathbf{y}(f)=\mathbf{H}(f) \mathbf{x}(f)+\mathbf{v}(f)
$$

When OFDM modulation is used, the entire channel is divided into a number of sub-channels. These sub-channels are spaced orthogonally to each other such that no inter-carrier interference (ICI) is present at the sub-carrier frequency subject to perfect sampling and carrier synchronization. When sampled at the subcarrier frequency of $f_{n_{c}}$, the channel model becomes.

$\mathbf{y}^{\left(n_{c}\right)}=\mathbf{H}^{\left(n_{c}\right)} \mathbf{x}^{\left(n_{c}\right)}+\mathbf{v}^{\left(n_{c}\right)} ; \quad n_{c}=-N_{c} / 2, \ldots ., N_{c} / 2-1$.

With $N_{c}$ sufficiently large, the sub-channel at each of the subcarriers can be regarded as flat-fading. Therefore, when using OFDM, the MIMO detection over frequency-selective channels is transformed into MIMO detection over $N_{c}$ narrowband flat-fading channels. For this reason, we only focus on the MIMO detection algorithms in flat-fading channels. The entries of the channel matrix $\mathbf{H}$ are assumed to be known to the receiver but not to the transmitter. This assumption is reasonable if training or pilot signals are sent to learn the channel, which is constant for some coherent interval.

\subsection{Problem Formulation}

The task is of detecting $N_{t}$ transmitted symbols from a set of $N_{r}$ observed symbols corrupted by a non-ideal communication channel, typically modeled as a linear system followed by an additive noise vector as shown in Figure 2.

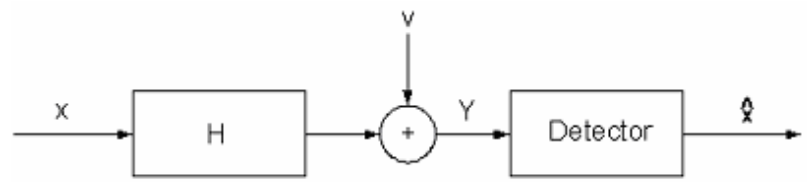

Figure 2: A simplified linear MIMO communication system showing the following discrete signals: transmitted symbol vector $x \in \chi^{N_{t}}$, channel matrix $\mathbf{H} \in \square{ }^{N_{t} x N_{r}}$, additive noise vector $\mathbf{V} \in \square^{N_{t}}$, receive vector $\mathbf{y} \in \square^{N_{t}}$, and detected symbol vector $\hat{S} \in \square{ }^{N_{r}}$. 
Transmitted symbols from a known finite alphabet $\chi=$ $\left\{x_{1}, \ldots, x_{M}\right\}$ of size $M$ are passed to the channel. The detector chooses one of the $M^{N t}$ possible transmitted symbol vectors from the available data. The optimal detector returns $\hat{\boldsymbol{x}}=x_{*}$, the symbol vector whose (aposteriori) probability of having been sent, given observed vector $\mathbf{y}$, is the largest:

$$
\begin{aligned}
\boldsymbol{x}_{*} \square & \underset{x \in \chi N_{t}}{\arg \max } P(\boldsymbol{x} \text { was sent } \mid \boldsymbol{y} \text { is observed }) \\
= & \underset{x \in \chi}{\arg \max _{t}} \frac{P(\boldsymbol{y} \text { is observed } \mid \boldsymbol{x} \text { was sent }) P(\boldsymbol{x} \text { was sent })}{P(\boldsymbol{y} \text { is observed })}
\end{aligned}
$$

This is known as Maximum Aposteriori Probability (MAP) detection rule. Making the standard assumption that the symbol vectors $\boldsymbol{x} \in \chi^{N_{t}}$ are equiprobable i.e. $P(\boldsymbol{x}$ was sent $)$ is constant, the MAP detector rule can be written as:

$$
\boldsymbol{x}_{*} \square \underset{x \in \chi}{\arg \max _{t}} P(\boldsymbol{y} \text { is observed } \mid \boldsymbol{x} \text { was sent })
$$

A detector that always returns an optimal solution satisfying (6) is called Maximum Likelihood (ML) detector. Assuming the additive noise $\mathbf{v}$ to be white and Gaussian, the ML detection problem of Figure 2 can be can be expressed as the minimization of the squared Euclidean distance to a target vector $\mathbf{y}$ over $N^{t}$ dimensional finite discrete search set:

$$
\boldsymbol{x}_{*}=\underset{x \in \chi}{\arg \min }\|\boldsymbol{y}-H \boldsymbol{x}\|^{2}
$$

Optimal ML detection scheme needs to examine all $M^{N t}$ or $2^{b N t}$ symbol combinations ( $b$ is the number of bits per symbol). It finds the $\mathbf{x}$ that minimizes the most likely transmitted symbol that causes the smallest difference (squared error) from the received signal. The problem can be solved by enumerating over all possible $\mathbf{x}$ and finding the one that causes the minimum value as in (7). Therefore, the computational complexity increases exponentially with constellation size $M$ and number of transmitters $N_{t}$.

We present a BPSO algorithm assisted wide band spatial multiplexing systems symbol detector that views the MIMO symbol detection issue as a combinatorial optimization problem and try to approximate the near optimal solution iteratively.

\section{SOME EXISTING MIMO DETECTORS}

\subsection{Linear MIMO Detectors}

A straightforward approach to recover $\mathrm{x}$ from y is to use an $N_{t} \mathrm{x} N_{r}$ weight matrix $\mathbf{W}$ to linearly combine the elements of $\mathbf{y}$ to estimate $\mathbf{x}$, i.e. $\hat{x}=\mathbf{W y}$.

\subsubsection{Zero-Forcing $(Z F)$}

The ZF algorithm attempts to null out the interference introduced from the matrix channel by directly inverting the channel with the weight matrix [10].

\subsubsection{Minimum Mean Squared Error (MMSE)}

A drawback of $\mathrm{ZF}$ is that nulling out the interference without considering the noise can boost up the noise power significantly, which in turn results in performance degradation. To solve this, MMSE minimizes the mean squared-error, i.e. $J(\mathbf{W})=\mathrm{E}\{(\mathrm{x}-\hat{x}$ )$\left.^{*}(\mathrm{x}-\hat{x})\right\}$, with respect to $\mathbf{W}[15],[16]$.

\subsection{Non-Linear MIMO Detectors}

\subsubsection{VBLAST}

A popular nonlinear combining approach is the vertical Bell labs layered space time algorithm (VBLAST) [11] This detection method is also called Ordered Successive Interference Cancellation (OSIC). It uses the detect-and-cancel strategy similar to that of decision-feedback equalizer. Either ZF or MMSE can be used for detecting the strongest signal component used for interference cancellation. The performance of this procedure is generally better than ZF and MMSE. VBLAST provides a suboptimal solution with lower computational complexity than ML. However, the performance of VBLAST is degraded due to error propagation.

\subsubsection{Detector}

Maximum Likelihood detector is optimal but computationally very expansive. ML detection is not practical in large MIMO systems.

\section{PSO FOR WIDE BAND SPATIAL MULTIPLEXING SYSTEM}

\subsection{Particle Swarm Optimization (PSO)}

Swarm intelligence argues that intelligent cognition derived from interactions of individuals in a social world and this sociocognitive approach can be effectively applied to computationally intelligent systems [5]. A swarm consists of a number of particles (possible solutions) that move (fly) through the feasible solution space to explore the optimal solution that can be coded as binary strings or real-valued vectors. The particles are capable of interacting with each other in a given neighborhood, and traverse a search space where a quality measure, fitness can be evaluated. The particles are evolved through cooperation and competition among themselves over iterations. The coordinates of each particle represent a possible solution associated with two vectors, the position $\left(\boldsymbol{X}_{i}\right)$ and velocity $\left(v_{i}\right)$. In d-dimensional search space, the $i^{\text {th }}$ particle can be represented by d-dimensional position vector $\boldsymbol{X}_{i}=\left(x_{i 1}, x_{i 2}, \ldots, x_{i d}\right)$ and another d-dimensional velocity vector $\boldsymbol{V}_{i}=\left(v_{i 1}, v_{i 2}, \ldots, v_{i d}\right)$. Each particle experiences an iterative procedure of adaptation to two types of major information i.e. individual learning and cultural transmission, which means the procedure, accelerates particles at each time step, towards personal best and the position of the most recent global best point, with the relative acceleration towards each determined stochastically. A key attractive feature of the PSO approach is its simple mathematical model involving two model equations [5].

In binary PSO [3], velocity loses its physical meaning. It is used to determine a probability by squashing velocities to the range $(0,1)$ by using sigmoid function. 


\subsection{PSO based MIMO-OFDM Detection.}

Here we exploit parsimonious binary choice PSO algorithm's potential to optimize MIMO symbol detection. An important step to implement PSO is to define a fitness function; this is the link between the optimization algorithm and the real world problem. Fitness function is unique for each optimization problem. The fitness function using the coordinates of the particle returns a fitness value to be assigned to the current location. If the value is greater than the value at respective personal best (pbest) for each particle, or global best (gbest) of the swarm, then previous locations are updated with the present locations. The velocity of the particle is changed according to the relative locations of pbest and gbest as shown in Figure 3.

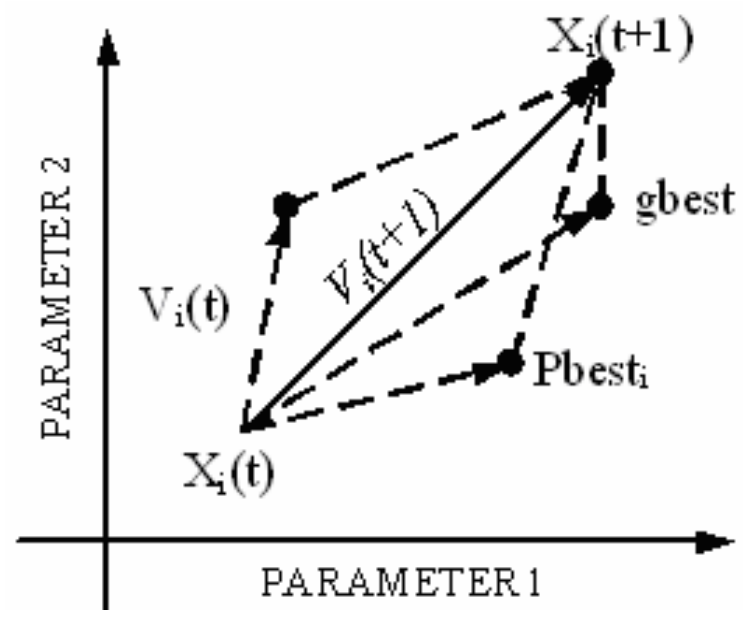

Figure 3.Vector representation of PSO model

Once the velocity of the particle is determined, it simply moves to the next position. After this process is applied on each particle in the swarm, it is repeated till the maximum number of iterations is reached. PSO algorithms flow diagram is shown in Figure 4. This exploratory-exploitive optimization approach can be extended to MIMO detection optimization problem discussed below.

The major challenge in designing Binary PSO based MIMOOFDM detector was selection of BPSO parameters that fit the symbol detection optimization problem. The basic fitness function used by the optimization algorithm to converge to the near optimal solution is (7) that is minimum Euclidian distance. Selection of initial guess is essential for these algorithms to perform. Therefore, our detector takes the output of ZF-VBLAST as its initial solution guess. This educated guess enables the algorithm to reach more refined solution iteratively by ensuring fast convergence. Assuming random initialization does not guarantee convergence to reasonable solution in lesser iterations.

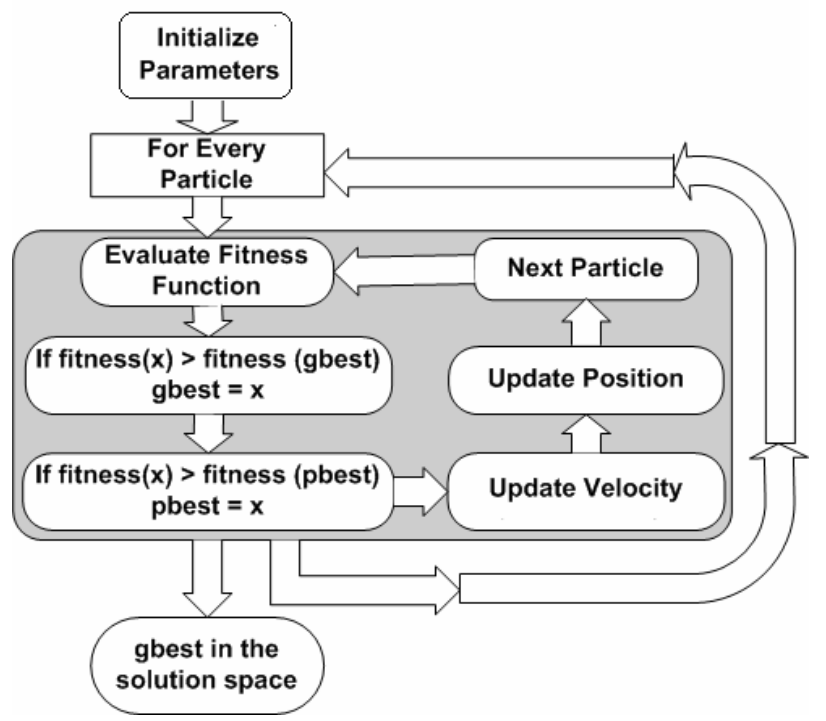

Figure 4. PSO flow diagram.

The proposed detection algorithm is detailed below:

1) Take the output of ZF-VBLAST such as $\mathbf{x}_{\mathbf{i}} \in\{0,1\}$ as initial particles (initial solution bit string) instead of selecting randomly from the solution space.

2) The algorithm parameters are initialized. ' $v_{\text {id }}$ ' is initialized to zero (equal probability for binary decision), 'pbest ${ }_{i d}$ ' and ' gbest $_{d}$ ' are initialized to maximum Euclidean distance depending upon the QAM size.

3) Evaluate the fitness of each particle (bit):

$$
f=\|\mathbf{y}-\mathbf{H x}\|^{2}
$$

Minimum Euclidean distance for each symbol represents the fitness of solution. Effect on the Euclidean distance due to search space bits is measured. Find the global best performance ' gbest $_{d}$ ' in the population that represents the least Euclidean distance found so far. Record the personal best 'pbest $t_{i d}$ ' for each bit along its previous values.

4) For each search space bit at $d^{\text {th }}$ side of the bit string of particle $\mathbf{x}_{\mathbf{i}}$, compute bits velocity using following PSO velocity update equation:

$$
\begin{aligned}
\mathrm{v}_{\mathrm{id}}(\mathrm{k}) & =\mathrm{v}_{\mathrm{id}}(\mathrm{k}-1)+\varphi_{1} \operatorname{rand}_{1}\left[\text { pbest }_{i d}-\mathrm{x}_{\mathrm{id}}(\mathrm{k}-1)\right] \\
& \left.+\varphi_{2} \text { rand }_{2} \text { gbest }_{d}-\mathrm{x}_{\mathrm{id}}(\mathrm{k}-1)\right]
\end{aligned}
$$

with $\mathrm{v}_{\mathrm{id}} \in\left\{-\mathrm{v}_{\max }, \mathrm{v}_{\max }\right\}$.

5) The particle position is updated depending on the following binary decision rule:

$$
\text { If } \operatorname{rand}_{3}<S\left(v_{i d}(k)\right) \text {, then } x_{i d}(k)=1 \text {, else } x_{i d}(k)=0 \text {. }
$$

6) Goto step 3 until maximum number of iterations is reached. The number of iterations is system and requirement dependent (usually kept less than 25 to avoid large complexity). Solution gets refined iteratively.

Here ' $\mathrm{k}$ ' is the number of iterations, rand is a random number generated uniformly in $[0,1]$ and ' $S$ ' is sigmoid transformation function. 


$$
S\left(v_{i d}(k)\right)=\frac{1}{1+\exp \left(-v_{i d}(k)\right)}
$$

The parameter ' $\mathrm{v}_{\mathrm{i}}$ ' is the particles predisposition to make 1 or 0 , it determines the probability threshold to make this choice. The individual is more likely to choose 1 for higher $v_{i d}(k)$, whereas its lower values will result in the choice of 0 . Such a threshold needs to stay in the range of $[0,1]$. The sigmoid logistic transformation function maps the value of $v_{i d}(k)$ to a range of $[0,1]$. The terms $\varphi_{I}$ and $\varphi_{2}$ are positive acceleration constants used to scale the contribution of cognitive and social components such that $\varphi_{1}+\varphi_{2}$ $<4$ [2]. These are used to stochastically vary the relative pull of pbest and gbest. $\mathrm{v}_{\max }$ sets a limit to further exploration after the particles have converged. Its values are problem dependent, usually set in the range of $[-4,+4]$.

\section{SIMULATION AND NUMERICAL RESULTS}

This section provides some simulation and numerical results to prove the performance of the reported MIMO-PS detector.

\subsection{BER versus SNR Performance}

We evaluate the PSO based MIMO detectors performance for a $3 \times 3\left(N_{r} x N_{r}\right), 4 \times 4,5 \times 5$ and 8x8 MIMO system using 4-QAM scheme. 128 sub-carriers and cyclic prefix of length 32 are used. The particle size (solution bit string length) ' $N_{p}$ ' dependents upon the QAM size and number of transmitters used in the spatial multiplexing system. $N_{p}=b \mathrm{x} N_{t}$ where ' $b$ ' is bits per symbol. For $3 \times 3$, 4-QAM system , ' $N_{p}$ ' equals 6 and it grows to 16 for $8 \times 8$, 4QAM system. ' $N_{i t r}$ ' is kept in the range of 5 to 20 in our simulations. Iterations can be tuned like other algorithm parameters according to the system requirements. Larger $N_{i t r}$ can result in achieving better BER performance at the cost of complexity. $\varphi_{1}=\varphi_{2}=1$ and $\mathrm{v}_{\max }= \pm 4$ are assumed in the simulations. An average of no less than 10,000 simulations is taken to report statistically relevant results.

The $S N R\left(\mathrm{E}_{\mathrm{b}} / \mathrm{N}_{\mathrm{o}}\right)$ is the average Signal to noise ratio per antenna $\left(\boldsymbol{P} / \boldsymbol{\sigma}_{v}^{2}\right)$, where $\boldsymbol{P}$ is the average power per antenna and $\boldsymbol{\sigma}_{v}^{2}$ is noise variance. The simulation environment assumes Rayleigh flat-fading channel with no correlation between sub-channels. Figure 5 present the bit error rate (BER) versus $\mathrm{E}_{\mathrm{b}} / \mathrm{N}_{\mathrm{o}}$ performance of proposed detector $\left(N_{i t r}=5\right)$ compared with ML and VBLAST detectors in $3 \times 3$ spatial multiplexing system. The proposed technique results in $3-\mathrm{dB}$ degraded performance at $10^{-4}$ BER in comparison with ML. However, in comparison to VBLAST, it shows 13-dB better performance.

In a 4x4, 4-QAM system shown in Figure 6, the performance gain compared to VBLAST is substantial. However, this BER gain is at a cost of increased computational complexity discussed in the next sub-section.

Similarly, for a $5 \times 5$ and 8x8, 4-QAM, MIMO-OFDM system given in Figure 7 and Figure 8, the proposed detection techniques gives improved BER performance as compared to VBLAST with some complexity overhead. The performance degrades by $4-\mathrm{dB}$ to $5-\mathrm{dB}$ comparative to optimal detector, but here the complexity gain is substantial (discussed next).

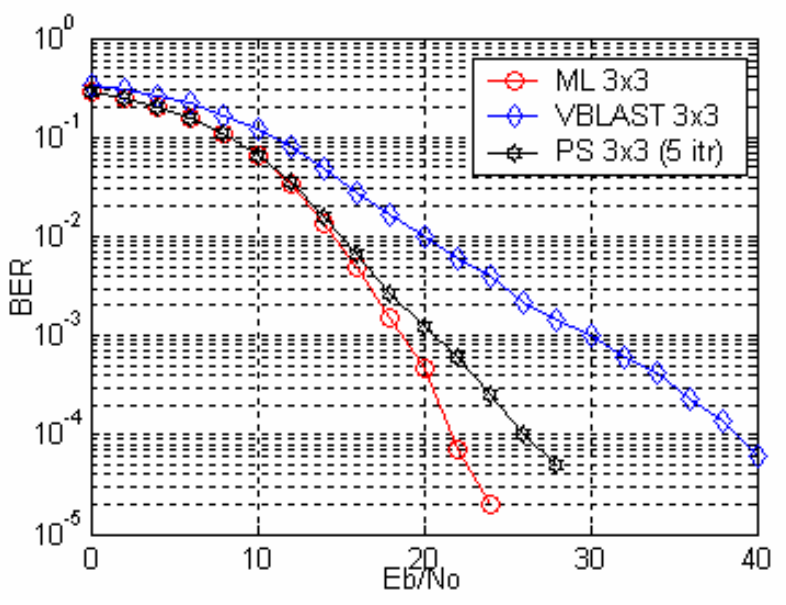

Fig. 5. BER versus $E_{b} / N_{0}$ for 4-QAM 3x3 MIMO system.

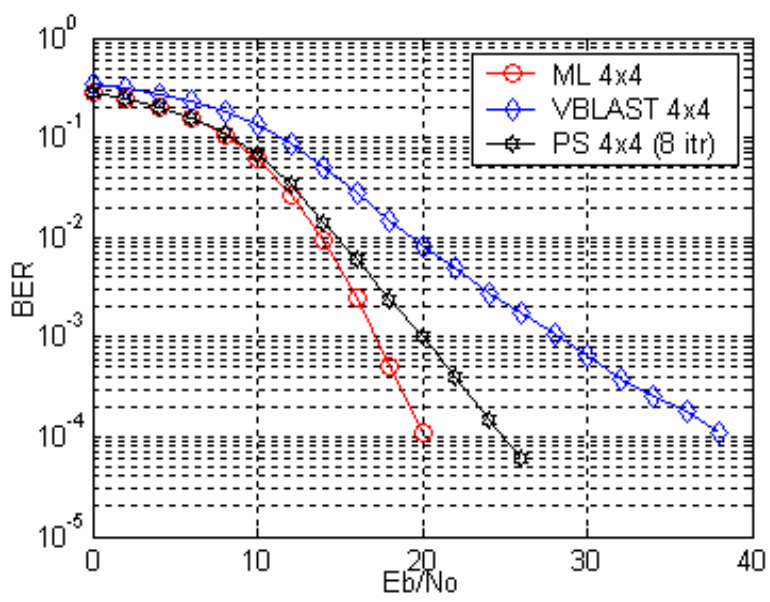

Fig. 6. BER versus $E_{b} / \mathbf{N}_{0}$ for $4-Q A M ~ 4 \times 4$ MIMO system.

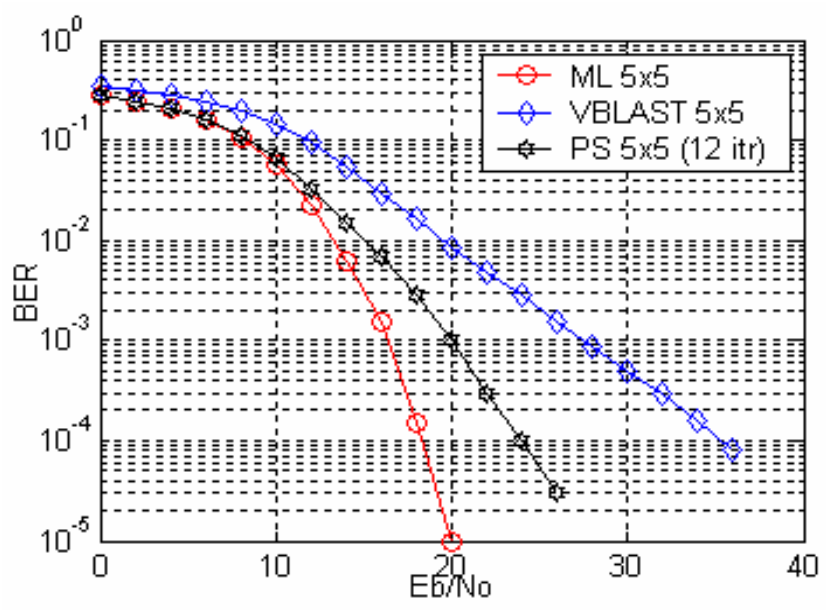

Fig. 7. BER versus $\mathbf{E}_{b} / \mathbf{N}_{0}$ for 4-QAM $5 \times 5$ MIMO system.

With the increase in the MIMO system complexity $\left(N_{t} x N_{r}\right)$, the size of search space also increase exponentially, therefore more algorithm iterations are required to reach near-optimal solution. A trade of between systems BER performance and iterations has to be maintained according to the system requirement and priority. 


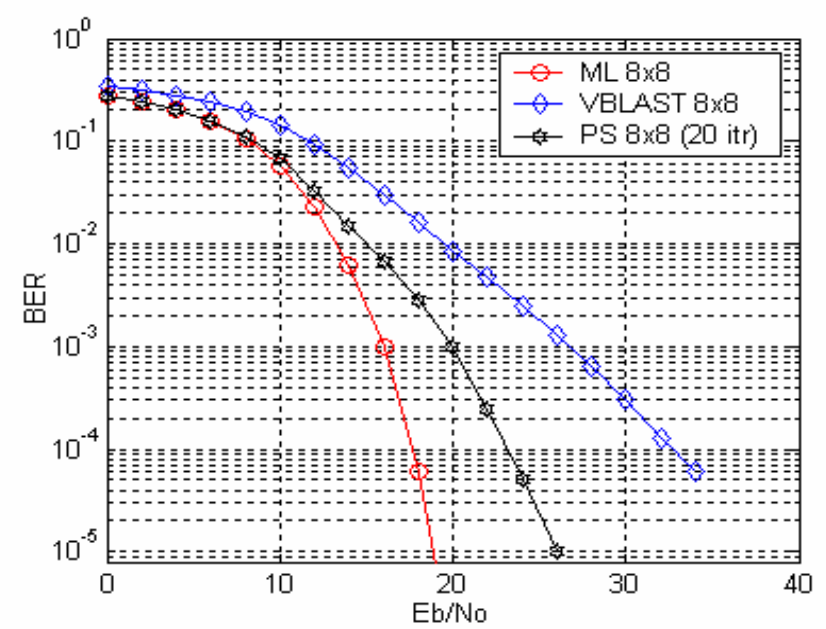

Fig. 8. BER versus $E_{b} / N_{0}$ for 4-QAM 8x8 MIMO system.

\subsection{Computational complexity comparison}

Here we examine the computational complexity of the reported PS detector and compare it with ML and VBLAST detectors. As the hardware cost of each algorithm is implementation-specific, we try to provide a rough estimate of complexity in terms of number of complex multiplications. The computational complexity is computed in terms of the $N_{t}, N_{r}$ and $M$ constellation size. ML detection requires $N_{r}\left(N_{t}+1\right) M^{N t}$. complex multiplications, as seen from (7). Where $M^{N t}\left(N_{r} N_{t}\right)$ is for matrix multiplication and $M^{N t} N_{r}$ is for square operation.

For VBLAST, the pseudo-inverse of matrix $\left(\mathbf{H}^{H} \mathbf{H}\right)^{-1} \mathbf{H}^{H}$ takes $4 N_{t}^{3}+2 N_{t}^{2} N_{r}$ multiplications [17]. The pseudo-inverse matrix is calculated $N_{t}$ times with decreasing dimension. In addition, the complexity of ordering and interference canceling is $\sum_{i=0}^{N_{t}-1}\left[N_{t}\left(N_{t}-i\right)+2 N_{t}\right]$. Therefore, total complexity of VBLAST $\left(\gamma_{\text {VBLAST }}\right)$ results in.

$$
\begin{aligned}
& =\sum_{i=0}^{N_{t}}\left(4 i^{3}+2 N_{r} i^{2}\right)+\sum_{i=0}^{N_{t}-1}\left[N_{t}\left(N_{t}-i\right)+2 N_{t}\right] \\
\gamma_{\text {VBLAST }} & =N_{t}^{4}+\left(\frac{5}{2}+\frac{2}{3} N_{r}\right) N_{t}^{3}+\left(\frac{7}{2}+N_{r}\right) N_{t}^{2}+\frac{1}{3} N_{t} N_{r}
\end{aligned}
$$

For the proposed detector, first fitness using (7) in the bit-string (particle size) ' $N_{p}$ ' is calculated. Multiplication complexity $\left(\gamma_{\mathrm{PS}}\right)$ becomes,

$$
\gamma_{P S}=N_{p}\left(N_{t} N_{r}\right)
$$

Velocity update for each particle requires $\mu_{v e l}$ additional multiplications per iteration with rand and $\varphi$ from (9). To reduce some complexity $\varphi_{1}+\varphi_{2}=2$ is assumed. Therefore $\mu_{v e l}$ becomes 4 , the complexity becomes,

$$
\gamma_{P S}=N_{p}\left(N_{t} N_{r}+\mu_{v e l}\right)
$$

This procedure is repeated $N_{\text {itr }}$ (number of iterations same as k) times to converge to the near-optimal BER performance. Therefore,

$$
\gamma_{P S}=N_{p}\left(N_{t} N_{r}+\mu_{v e l}\right) N_{i t r}
$$

Since the Proposed detector takes initial solution guess as the output of ZF-VBLAST therefore, total computational complexity of proposed detector results in,

$$
\gamma_{P S_{\text {tatal }}}=\gamma_{P S}+\gamma_{V B L A S T}
$$

The complexity of the proposed method compared with ML and VBLAST detectors for several configurations is presented in Table-1. The complexity estimate in Table-1 is only meaningful in the order of magnitude sense since it is based on the number of complex multiplications. The above complexity is estimated on subcarrier-by-subcarrier for MIMO-OFDM system.

\subsection{Performance-complexity trade-off.}

Results in Table-2, show that a reasonable performancecomplexity trade-off exists when a comparison of the proposed detector is drawn with ML and VBLAST detectors.

Compared with ML the complexity reduction of the proposed detector is significant for larger MIMO systems where ML is not practical to use. However, this complexity gain is at the cost of degraded BER performance. For a $5 \times 5$, 4-QAM system, at $10^{-4}$ BER the performance of proposed detector is degraded by 4-dB, with $83 \%$ complexity reduction. Similarly, in 8x8, 4-QAM system, the proposed algorithm $\left(N_{i t r}=20\right)$ achieves $10^{-4}$ BER at 5$\mathrm{dB}$ more SNR than ML. However, the ML complexity reduction is $99 \%$.

When compared with VBLAST the proposed detector complexity increase approximately $70 \%$ with a BER gain up to $12-\mathrm{dB}$ for larger systems.

\section{CONCLUSIONS}

In this contribution, application of Binary PSO algorithm for symbol detection in spatial multiplexing system is reported. PSO,s simple mathematical model, lesser implementation complexity, resistance to being trapped in local minima and convergence to reasonable solution in lesser iterations makes it a suitable candidate for real-time wireless communications systems. This algorithm shows promising results when compared with the optimal ML and traditional VBLAST detectors. Particle swarm intelligence optimized MIMO symbol detection mechanism approaches near-optimal performance with much reduced computational complexity, especially for complex systems with multiple transmitting antennas, where conventional ML detector is computationally expensive and impractical to deploy. Although VBLAST detector has a reduced complexity, its BER performance is inferior to the proposed detector. The simulation results suggest that the proposed detector improves ML complexity by as high as $99 \%$ with 5 -dB BER performance degradation in a 8x8 MIMO system.

\section{REFERENCES}

[1] J. Kennedy and R. C. Eberhart, "Particle Swarm Optimization," in Proc. IEEE International Conference on Neural Networks IV, Piscataway, NJ, 1995.

[2] J. Kennedy and R. C. Eberhart, Swarm intelligence, Morgan Kaufman publisher, 2001. 
[3] J. Kennedy and R. C. Eberhart, "A discrete binary version of the particle swarm algorithm," IEEE International Conference on Systems, Man, and Cybernetics, pp.4104, 1997.

[4] H. H. El-Mora, A.U. Sheikh and A. Zerguine, "Application of Particle Swarm Optimization Algorithm to Multiuser Detection in CDMA", $16^{\text {th }}$ IEEE International Symposium on Personal, Indoor and Mobile Radio communications". 2005.

[5] R.C. Eberhart and Y. Shi, "Particle Swarm Optimization: developments, applications and resources", IEEE, 2001

[6] A.Goldsmith, S.A. Jafar, N.Jindal, and S. Vishwanath, "Capacity limits of MIMO channels", IEEE Journal on Selected Areas in Communications, Vol 20, No 5, June 2003.

[7] Syed Aon Mujtaba. (2005, May). TGn Sync Proposal Technical Specification. Agere Systems Inc., U.S.A. [Online]. Available: http://www.tgnsync.org/techdocs/11-040889-06-000n-tgnsyncproposal-technical-specification.doc

[8] L.Hanzo, Münster, B. Choi, and T. Keller, OFDM and $M C$ CDMA for Broadband Multi-user Communications, WLANs and Broadcasting. John Wiley and IEEE press, 2003.

[9] L. Zheng and D. Tse, "Diversity and multiplexing: a fundamental tradeoff in multiple antenna channels," IEEE Tran. Inform. Th., vol. 49, pp. 1073-96, May 2003.

[10] H. Bolcskei, D. Gesbert, C. and Papadias, Space-Time Wireless Systems: From Array Processing to MIMO Communications, Cambridge University Press, 2005.

[11] G. J. Foschini, "Layered space-time architecture for wireless communication in a fading environment when using multiple antennas," Bell Labs Technical Journal, vol. 1, pp. 41-59, Autumn 1996.

[12] G. J. Foschini and M. J. Gans, "On limits of wireless communications in a fading environment when using multiple antennas," Wireless Personal Communications, 1998, Vol. 6, No.3, pp. 311-335.

[13] W. J. Choi, R. Negi and J. M. Cioffi, "Combined ML and DFE decoding for the V-BLAST system," in Proc. IEEE International Conference on Communications 2000, New Orleans, LA, Jun. 2000. pp. 1243-1248.

[14] W. J. Choi, K. W. Cheong and J. M. Cioffi, "Iterative soft interference cancellation for multiple antenna systems," in Proc.IEEE Wireless Communications and Networking Conference 2000, Chicago, IL, Sep. 2000. pp. 304-309.

[15] S. Haykin, Adaptive Filter Theory, 3rd ed. Prentice-Hall, 1996.

[16] A. Sayed, Fundamentals of Adaptive Filtering. Wiley-IEEE Press, 2003.

[17] G. H. Golub and C. F. V. Loan, Matrix Computations, 3rd ed. John Hopkins University Press, 1996.

TABLE 1,

COMPLEXITY COMPARISON (Complex Multiplications) - 4QAM $N_{r} x N_{r}$ MIMO system

\begin{tabular}{|c|c|c|c|c|}
\hline Method & $\mathbf{3 x 3}$ & $\mathbf{4 x 4}$ & $\mathbf{5 x 5}$ & $\mathbf{8 x 8}$ \\
\hline ML $\left(\gamma_{M L}\right)$ & 768 & 5120 & 30720 & $4.7 \mathrm{M}$ \\
\hline VBLAST $\left(\gamma_{V B L A S T}\right)$ & 265 & 712 & 1572 & 8864 \\
\hline Proposed & $\left(N_{p}=6, N_{i t r}=5\right.$, & $\left(N_{p}=8, N_{i t r}=8\right.$, & $\left(N_{p}=10, N_{i t r}=12\right.$, & $\left(N_{p}=16, N_{i t r}=20\right.$, \\
PS detector $\left(\gamma_{P S_{\text {tatal }}}\right)$ & $\left.\mu_{v e l}=4\right)$ & $\left.\mu_{v e l}=4\right)$ & $\left.\mu_{v e l}=4\right)$ & $\left.\mu_{v e l}=4\right)$ \\
& 655 & 1992 & 5052 & 30624 \\
\hline
\end{tabular}

TABLE 2,

PERFORMANCE COMPLEXITY TRADE-OFF - 4QAM $N_{r} x N_{r}$ MIMO system

\begin{tabular}{|c|c|c|c|c|c|}
\hline \multicolumn{2}{|c|}{ Performance complexity comparison } & $3 \times 3$ & $4 \times 4$ & $5 \times 5$ & $8 \times 8$ \\
\hline \multirow{2}{*}{$\begin{array}{l}\text { ML and } \\
\text { proposed } \\
\text { detector }\end{array}$} & $\begin{array}{l}\text { Complexity reduction } \\
\qquad\left(\gamma_{M L}-\gamma_{P S_{\text {tatal }}}\right) / \gamma_{M L}\end{array}$ & $14 \%$ & $61 \%$ & $83 \%$ & $99 \%$ \\
\hline & $\begin{array}{c}\text { Performance degradation } \\
\text { at } 10^{-4} \mathrm{BER} \\
\text { (approximately) }\end{array}$ & $3 \mathrm{~dB}$ & $3 \mathrm{~dB}$ & $4 \mathrm{~dB}$ & $5 \mathrm{~dB}$ \\
\hline \multirow{2}{*}{$\begin{array}{c}\text { VBLAST and } \\
\text { proposed } \\
\text { detector }\end{array}$} & $\begin{array}{l}\text { Complexity increase } \\
\left(\gamma_{P S_{\text {tatal }}}-\gamma_{V B L A S T}\right) / \gamma_{P_{\text {total }}}\end{array}$ & $59 \%$ & $64 \%$ & $68 \%$ & $71 \%$ \\
\hline & $\begin{array}{c}\text { Performance } \\
\text { improvement at } 10^{-4} \\
\text { BER (approximately) }\end{array}$ & $13 \mathrm{~dB}$ & $12 \mathrm{~dB}$ & $12 \mathrm{~dB}$ & $10 \mathrm{~dB}$ \\
\hline
\end{tabular}

